\title{
Neural Computing for Scientific Computing Applications
}

\author{
More than Just Machine Learning
}

\author{
James B. Aimone \\ Center for Computing Research \\ Sandia National Laboratories \\ P.O. Box 5800 \\ Albuquerque, New Mexico 87185-1327 \\ jbaimon@sandia.gov
}

\author{
Ojas Parekh \\ Center for Computing Research \\ Sandia National Laboratories \\ P.O. Box 5800 \\ Albuquerque, New Mexico 87185-1327 \\ odparek@sandia.gov
}

\author{
William Severa \\ Center for Computing Research \\ Sandia National Laboratories \\ P.O. Box 5800 \\ Albuquerque, New Mexico 87185-1327 \\ wmsever@sandia.gov
}

\begin{abstract}
Neural computing has been identified as a computing alternative in the post-Moore's Law era, however much of its attention has been directed at specialized applications such as machine learning. For scientific computing applications, particularly those that of ten depend on supercomputing, it is not clear that neural machine learning is the exclusive contribution to be made by neuromorphic platforms. In our presentation, we will discuss ways that looking to the brain as a whole and neurons specifically can provide new sources for inspiration for computing beyond current machine learning applications. Particularly for scientific computing, where approximate methods for computation introduce additional challenges, the development of non-approximate methods for neural computation is potentially quite valuable. In addition, the brain's dramatic ability to utilize context at many different scales and incorporate information from many different modalities is a capability currently poorly realized by neural machine learning approaches yet offers considerable potential impact on scientific applications.
\end{abstract}

\section{CCS CONCEPTS}

- Computer systems organization $\rightarrow$ Neural networks; $\bullet$ Computing methodologies $\rightarrow$ Parallel algorithms;

\section{KEYWORDS}

Neuromorphic Computing, Scientific Computing, High Performance Computing, Brain

\section{ACM Reference format:}

James B. Aimone, Ojas Parekh, and William Severa. 2017. Neural Computing for Scientific Computing Applications. In Proceedings of Neuromorphic Computing Symposium, Knoxville, TN, USA, fuly 17-19, 2017 (NCS '17), 6 pages. https://doi.org/10.1145/3183584.3183618

ACM acknowledges that this contribution was authored or co-authored by an em ployee, or contractor of the national government. As such, the Government retains a nonexclusive, royalty-free right to publish or reproduce this article, or to allow others to do so, for Government purposes only. Permission to make digital or hard copies for personal or classroom use is granted. Copies must bear this notice and the full citation on the first page. Copyrights for components of this work owned by others than ACM must be honored. To copy otherwise, distribute, republish, or post, requires prior specific permission and/or a fee. Request permissions from permissions@acm.org. NCS '17, fuly 17-19, 2017, Knoxville, TN, USA

(C) 2017 Association for Computing Machinery.

ACM ISBN 978-1-4503-6442-3/17/07 . \$ \$15.00

https://doi.org/10.1145/3183584.3183618

\section{INTRODUCTION}

Neural computation is often touted as one of the more promising technology paths for computing beyond Moore's Law [6]. While the brain has been cited as an inspiration for computing for generations, recent advances in neuromorphic hardware platforms (such as IBM TrueNorth $[12,25]$ ) and neural machine learning algorithms (such as deep learning [18]) have renewed interest in neural computation. Although both algorithmic and hardware solutions inspired by the brain have disruptive potential, the field is still in its infancy and there seemingly are as many proposed approaches as there are individuals pursuing it. For this reason, neural computing needs to be motivated by applications as it is not yet clear if a universal neural computer can or should exist.

There are a number of challenges related to scientific computing that neural approaches may be able to impact, particularly within applications that typically require high performance computing (HPC), which is an unexplored field for neural computing methods and platforms. These include energy consumption, parallelizability, communication, and robustness. However, in order to effectively leverage these generally perceived advantages, it is crucial to ask whether neural approaches have real advantages for scientific computation at large scale (either theoretically or practically) and, if so, what is the best route to integrate those capabilities into computing design. Taking a rigorous approach is useful because of the counterarguments against using neural approaches for scientific applications, such as the perceived low precision of cognitive functions, the large degree of interconnectivity within the brain, and lack of precise algorithmic descriptions of brain functionality.

One of the major challenges to leveraging neural inspiration for applications such as this is the relative vagueness that typically accompanies the brain as a motivation for computing. There are many aspects of biological neural computation that may be useful for new computing approaches, and likewise there are characteristics of the brain that are perhaps less desirable for particular applications. Because most neuromorphic computing efforts look to the brain as inspiration as opposed to a concrete blueprint, it is important to recognize that we can leverage some aspects of biology while ignoring others. This ability to select a subset of neural characteristics for inspiration implies that neuromorphic computing is not best represented by a single, common universal model, but rather is best thought of as a family of related models with different areas of emphasis.

In this paper, we describe two distinct models of neural computation that leverage different aspects of the brain for inspiration. While we leave for future work extensive formal analysis of these 
models, we sufficiently formalize them so as to highlight the relative advantages and differences of each approach.

If neuromorphic computing is to have a deep, scalable impact on computing broadly [1], we contend that it is crucial to identify areas where it can impact the core goals of scientific computation as opposed to simply making inroads on the periphery of areas of impact. As with many emerging technologies, there are a number of distinct approaches for neural computing to best impact a postMoore's law environment, and each of these approaches comes with different benefits and challenges. Here, we will briefly overview how two of these distinct perspectives can impact the challenges facing scientific computing today, and given our stated goal, propose a strategy by which neural computing can lead to impacts at both the relative near-term and over the foreseeable future.

\section{NEURAL INSPIRATION FOR COMPUTING IMPACT}

While the brain has been seen as an intriguing source of inspiration for computing since the early days of the field [36, 41], it has long been challenging to be concrete about what aspects of neuroscience are most useful for the computing field. The challenging integration of the physical, biological system and the processing of information requires a distinction between mechanisms and computation. Thus, an important challenge in formulating models of neural computation is differentiating between computational properties that a neural computer should achieve and neurobiological mechanisms that we may choose to emulate in order to achieve those performance characteristics.

Here, we outline several different properties of biological neural systems that provide potential outcomes of a brain-inspired model. This is by no means an exhaustive list of potential desirable or notable properties, and none of these are universal across neural systems. Further, not all of these may be considered 'positive' for engineering purposes, however they are worth noting nonetheless.

Shallow-depth: In most biological neural systems, sensory information can induce highly sophisticated responses in very short physiological time. While the extensive levels of recurrence in the brain complicates the computing notion of depth, this observation suggests that the brain can process information in very few processing steps. This is quite distinct from the highly iterative approaches used by serial computing and different from all but the simplest deep artificial neural networks.

Rapid, stable learning: While it differs from system to system, mammalian brains are often capable of dynamically adjusting their internal representations in response to new information from the world, thus satisfying the stability-plasticity dilemma that often plagues conventional machine learning approaches.

High relational memory capacity: The absolute capacity of neural systems is still an area of ongoing study [40]. However, practical experience suggests that the effective capacity is large. The contrast here between biology and technology is stark as the storing and accessing of large relational databases is still a common modern computing challenge.

Context-enriched processing: Many neural circuits are able to dynamically adjust how information is processed based on what is generally considered contextual information, which could involve considering previous experiences in a decision, incorporating peripheral sensory information in processing, or biasing a behavior based on internal state.

Low-power: That the brain is low power is often cited as a motivation for neuromorphic computing [10], however the complex functions and biophysics of neural systems makes the attribution of the brain's low power consumption an ongoing challenge.

Unreliable and noisy: While the extent that the brain is stochastic can be debated, biological neural systems are typically observed to be less reliable than engineered systems. Regardless, distributed representations can allow for robust neural computation.

Poor numerical capabilities: While neural circuits can learn to perform highly complex tasks, many of which require sophisticated numerical solutions to solve conventionally, it is clear that the human brain is not optimized for high-precision numerical calculations.

\section{EXPLICIT MODELS OF NEURAL COMPUTATION}

Given the above properties that a neural computer may emulate, the next step in defining a model of neural computation is to identify different biological mechanisms. These models, in turn, are defined by different biological mechanisms that are commensurate with the scale from which neural inspiration is taken.

Here, we describe two different models for neural computation that illustrate how taking inspiration from the brain at different scales can yield dramatically different impacts on computing. The first model, the spiking threshold gate model, focuses on neurons as a mechanism and enables numerical computing as an application. This model is the most mature, as it is both clearly definable and the focus of neuromorphic architectures today. We follow by describing a higher level cognitive model, one focused on the neural circuit architecture of the brain as opposed to the individual neuron components. This perspective of neural computation enables a very different set of capabilities and considerations for utilizing in computing applications.

\subsection{The Spiking Neural Threshold Gate Model}

For the first model, we select a level of inspiration most naturally compatible with the numerous neuromorphic hardware systems being developed [13, 28]. Specifically, the spiking neural threshold gate (SNTG) model leverages three sources of inspiration from the low-level processing of most neurons: the analog summation of inputs from upstream neurons, the non-linear generation of a 'spike' event that represents the sole communication to downstream neurons, and a maintenance of internal state from one time to the next. This model is similar to the leaky integrate-and-fire (LIF) neural model common in theoretical neuroscience.

This model allows us to consider what circuits of neurons can do generally, setting aside constraints imposed by biological neural circuits. While the brain uses very specific assemblies of neurons to perform very sophisticated cognitive computations; neurons are potentially very powerful devices for computation, with their high degree of inputs, non-linear processing, spike-based communication, and ability to adapt over time all potential sources of computing value. Here, we consider one of the more formally well-understood 
classes of neural computing, threshold gate (TG) circuits, and then ask how we can extend this work towards neuromorphic computing applications.

In addition to its relationship to neuromorphic hardware, the level of abstraction in this model also conveniently allows us to relate neural computation to the well established field of TG circuits, an approach of computer science that has its roots in McCullochPitts logic from the 1940s. TGs are known to be more powerful than conventional Boolean logic gates (e.g., AND, OR, NOT), and circuits composed of TGs are capable of performing a large number of sophisticated numerical operations, such as matrix multiplication, in constant depth and polynomial size with respect to inputs [32]. This is an interesting parallel to the brain, which is able to perform very sophisticated computations while at fixed size. Importantly, these TG circuits can often be configured, with some trade-off with space (number of nodes) or time, to achieve a desired level of precision.

For decades, theoretical computer science has studied TG circuits [30,33]. These circuits are directed acyclic graphs consisting of logic gates which are essentially threshold-activated perceptrons. That is, for a tuple of inputs $\left(x_{1}, \ldots, x_{k}\right)$ (where $x_{i} \in\{0,1\}$ ), a tuple of weights $\left(w_{1}, \ldots w_{k}\right)$ and a threshold $T$, the TG computes

$$
y\left(x_{1}, \ldots, x_{k}\right)=\left\{\begin{array}{l}
1, \sum_{i=1}^{k} w_{i} x_{i} \geq T \\
0, \text { otherwise }
\end{array}\right.
$$

The existence and interest in TGs as fundamental logical units helps motivate the use of neurons as fundamental computational units. A key difference, however, is the emergence of specialty neuromorphic hardware. These theoretical TGs act as special cases of LIF neurons implemented on neural computing hardware such as the IBM TrueNorth chip [25]. The prototypical neurons communicate a 1 or a "spike" if an internal decaying variable rises above a pre-determined threshold. If one considers that a classic TG neuron is effectively a LIF neuron without its dynamical time component, it is reasonable to then consider that a hardware platform designed for configurable LIF neuron architectures can theoretically implement anything that TG networks could accomplish.

There are several paths to realizing advanced neural functionality through neural-based logic, which is increasingly feasible with new hardware technologies $[15,25]$. One approach is to develop a neural accelerator, or co-processor, that utilizes neural circuits to perform otherwise costly functions. We believe a number of TG-inspired kernels could be produced - for instance enabling the low-power acceleration of large linear algebra operations and these could have a considerable impact if well integrated with existing processors.

3.1.1 TGs can compute complex numerical algebraic functions. TG circuits as a model of computation are equivalent to circuits of conventional Boolean gates with at worst a polynomial overhead in the number of gates. Thus they are capable of universal computation. To render such a theoretical equivalence practically viable, understanding resource advantages threshold circuits might provide over conventional logic circuits is paramount. Two critical resources are (i) the total number of gates in a circuit computing a given function of interest, and (ii) the depth of the circuit. The former corresponds to hardware resources while the latter corresponds to computation time. Energy is another critical resource, and Uchizawa, Douglas, and Maass have initiated the examination of tradeoffs between energy expenditure and other resources in threshold circuits [37-39]. Other more biologically motivated resource measures have also been proposed [19].

There are elementary TG circuits that can compute the product of two $n \times n$ matrices, with entries of magnitude $O(n)$, in constant depth using $\widetilde{O}\left(n^{3}\right)$ gates (the $\widetilde{O}$ notation ignores log factors). This may be compared with conventional Boolean circuits for computing the product of such matrices based on the standard textbook matrix multiplication algorithm. Such an approach yields a Boolean circuit of $O(\log n)$ depth using $\widetilde{O}\left(n^{3}\right)$ gates. This type of circuit may be viewed as a conventional parallel algorithm to multiply matrices in $O(\log n)$ time with $\widetilde{O}\left(n^{3}\right)$ total work. However, this can be improved; Strassen's famous matrix multiplication algorithm gives rise to a conventional $O(\log n)$-time parallel algorithm requiring only $\widetilde{O}\left(n^{\omega}\right)$ total work, for $\omega \approx 2.81$ [35]. Recent advances have yielded algorithms with $\omega \approx 2.37$ [5, 42], and it is a major open question whether an algorithm with $\omega=2$ is possible (this is the best possible since the input can be of size $\Omega\left(n^{2}\right)$ ).

These so-called fast matrix multiplication algorithms improve the total work, or hardware resource expenditure, while still taking $O(\log n)$ time. In contrast, TG circuits offer an avenue to constanttime algorithms for matrix multiplication. In recent work we have shown that any conventional $\widetilde{O}\left(n^{\omega}\right)$-work fast matrix multiplication algorithm may be converted to a constant-depth threshold circuit also requiring approximately $\widetilde{O}\left(n^{\omega}\right)$ gates [27]. Work-efficient constant-time parallel algorithms are extremely rare and are in some sense 'perfect' parallel algorithms. This is surprising since, although threshold circuits are polynomially equivalent to conventional Boolean circuits, they can offer substantial advantages for fundamental computational primitives. There are functions that polynomial-size threshold circuits can compute in constant depth that polynomial-size Boolean logic circuits cannot compute in constant depth [8, 43], and it is possible matrix multiplication is another such example.

From this perspective, threshold circuits are provably more powerful than Boolean logic circuits, and it is open whether such theoretical advantages may be leveraged to yield practical improvements. Work is currently underway to see if these new threshold circuit constructions can yield improved practical approaches to matrix multiplication and other scientific computing primitives by employing near-term neuromorphic hardware.

3.1.2 LIF neurons as enhanced TGs. While superficially the similarities between LIF neurons and TGs are numerous, there is a more formal relationship between spiking neurons and TGs that is worth considering as the field progresses. LIF neurons, of course, operate with a time dimension whereas TGs do not. Regardless the two models are equivalent in some sense, and thus we consider them together in our SNTG model. LIFs can readily simulate TGs, and circuits consisting of TGs may be used to simulate the dynamics of LIFs. The following practical primer is more illustrative.

By setting a LIF neuron to decay completely each time step, a TG is formed. Thus, as expected, replicating the behavior of a 
TG using a LIF neuron is trivial. Adding a time dimension to a TG in the objective of replicating a LIF neuron is only slightly more difficult. Consider a neuron $y$ that receives inputs $x_{i}$ each weighted $w_{i}$ and decays by a factor of $\lambda$ at each time step. We can replicate this neuron using a TG circuit pictured in Figure 1. Here, the activation of $y\left(t_{i}\right)$ is calculated in a collection of gates (grey) with $t_{i}$ corresponding to the depth of the particular gate. State information is propagated by various auxiliary gates (yellow) dependent on the activation of $y$ at the previous step; the node fires if and only if $x_{i}\left(t_{0}\right)-y\left(t_{1}\right)=1$. Two time steps are pictured, though the process can be extended indefinitely. Notice that delay is not incorporated as multiple LIF neurons can be chained effectively adding variable delay to a system with constant delay synapses.

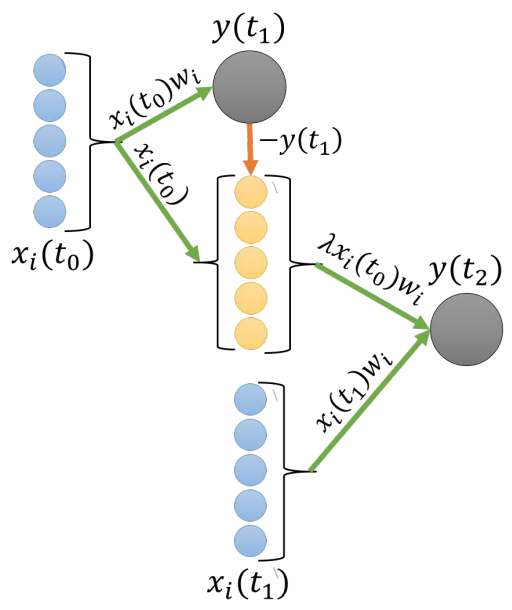

Figure 1: A circuit diagram wherein threhold gate units replicate the behavior of a leaky-integrate-and-fire neuron.

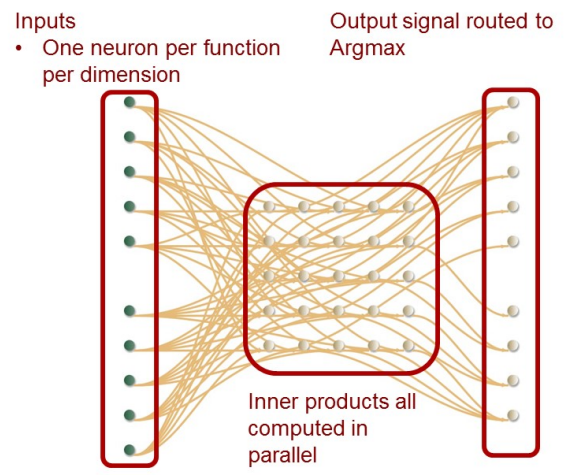

Figure 2: Example neural circuit for spiking cross correlation algorithm. Figure adapted from [29].

Importantly, the inclusion new neural-inspired primitives in the SNTG model, such as spiking, but also including temporal coding and recurrent connectivity, could extend neural computation beyond what is typically examined in TG logic. For instance, SNTG nodes can thought of as powerful resources that offer a nontrivial means of effeciently performing tasks typically considered for more conventional computing $[20,21]$. We have shown recently that basic scientific computational kernels, such as cross-correlations, are not only feasible with SNTGs, but provide computationally efficient trade-offs if using appropriate hardware [29]. Independently, Lagorce and Benosman developed a spiking-neuron-centric computation kernel useful for solving differential equations [16]. As with the TG argument above, we are confident that SNTG algorithms can be produced for many different basic computational functions that underlie scientific computing, and if appropriate neuromorphic hardware is used, these approaches can be highly energy-efficient.

One interesting facet of the spiking cross-correlation approach is the time-space trade off. Namely, while the overall complexity (i.e., total work) is fixed, the circuit can be designed to fit the required constraints and given resources. As an example, the circuit referenced above that computes the cross-correlation function in $O(n)$ time with $O(n)$ neurons can be re-structured to terminate in $O(1)$ time, but at the cost of $O\left(n^{2}\right)$ neurons.

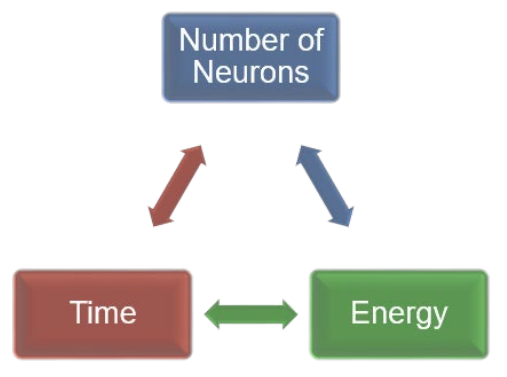

Figure 3: The temporal and parallel dimensions leveraged by spiking neural algorithms enable applications to prioritize time-, power-, or space-efficient formulations.

\subsection{Cortico-hippocampal neural circuit model}

While we have described extensively how a neural computing model focused at the neuron level can enable numerical computation, it is worth illustrating the value of higher level neural computing models. Specifically, models that emphasize features of the brain's neural circuit architecture can yield considerably different characteristics than the aforementioned spiking neuron model. Importantly, while the neuron level model we described above is reasonably genericmost neuromorphic architectures today are compatible with the above description-models based on higher level brain structures are likely far more specialized. In addition, these more cognitive models can vary widely in both scale and scope, including models loosely inspired by visual cortex such as deep ANNs or highly abstract cognitive frameworks [3, 4, 7, 17, 18].

Here, we illustrate a model of neural computation that is based on the higher level regional organization of the brain. While many cognitive functions are at first glance distinct from those which typically drive computing technologies; the brain as a whole, and in particular they way these circuit components interact with one another, offers a number of unique features that could be impactful. While the brain can thus offer intriguing sources of inspiration 
for revolutionary neural computing [1], the biggest challenge with looking exclusively at the neural circuits of the brain is that such an impact is likely a number of years away. Knowledge of biological neural circuits is rapidly progressing. Specifically the US BRAIN Initiative [2] and the EU Human Brain Project [22] are producing exciting new insights into neuroscience; however there is much remaining to be learned. Likewise, as the curious example above shows, the brain's approach to computation can be so different from conventional approaches it is not clear where to start making inroads.

Despite this relatively long outlook, there are already several possible opportunities for deeper brain inspiration to start influencing computing; particularly in areas that computing systems are being reassessed.

3.2.1 Brain-like memory to improve computing architectures. One example of how the brain can provide entirely new architectural inspiration to computing platforms is the brain's management of memory and computation. It is often noted that the brain colocalizes processing and memory at a number of scales-a feature that can offer clear advantages in a computing domain. For instance, at a system level, the cortico-hippocampal loop provides an interesting contrast to conventional architectures [24][34]. Short-term memories are formed and temporarily stored in the hippocampus region, which is believed to act more like an adaptive contentaddressable memory (CAM) [23]. The cortex, generally believed to be the primary site of "CPU-like" processing of incoming sensory information and outgoing motor decisions, also serves as the site of long-term data storage [31]. While both CAMs and processing in memory architectures are often discussed as future computing technologies (see for instance the "Neural Turing Machine" by Google DeepMind [9]), the concept of putting the processing on the metaphorical hard drive cleanly separated from the far faster RAM is quite unusual and counter-intuitive, and likely an approach that benefits from the specific parallel implementation in the brain.

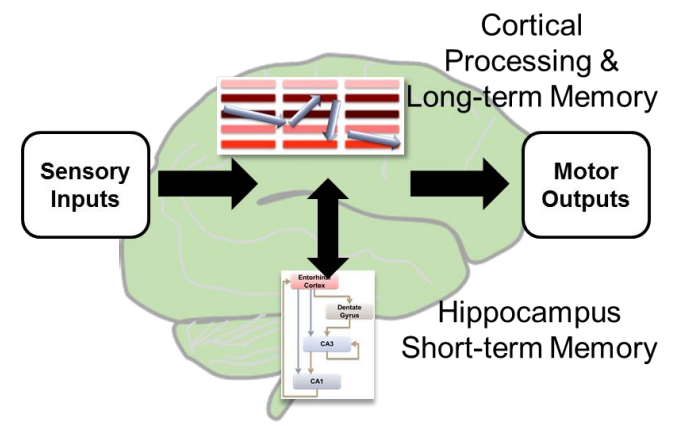

Figure 4: Relationship of cortex and hippocampal structures in brain processing.

Importantly, such a neural CAM potentially would be quite distinct from the more conventional CAMs seen today in systems such as network switches [26]. A hippocampus-inspired CAM would likely look more similar to Hopfield networks [11] and other recurrent memory networks that were studied extensively in the 1980 s than conventional CAMs such as hardware-implemented associative arrays. However, while Hopfield and other similar networks, such as sparse distributed memories [14], offer compelling features such as a clear neural formulation and auto-associative memory retrieval, they are fairly restricted in terms of being limited to a sublinear effective capacity and being generally difficult to gracefully adapt to new inputs.

In contrast, the hippocampus, the brain's principal associative memory structure, is likely more powerful on both dimensions. Regarding adaptivity, the hippocampus has long been believed to be responsible for rapid, or "one-shot," learning of declarative information (i.e., facts such as "Paris is the capital of France" and experiences such as "I had blackberry pie while visiting my aunt last week"). Humans who have had clinical lesions to their hippocampi have profound deficits in forming such new memories, yet pre-injury experiences and knowledge can often be retrieved. Regarding capacity, it is not immediately clear what the hippocampus's memory capacity is; however, it is notable that the cortex neuron numbers scale much faster than the hippocampus as brain size increases, suggesting that the hippocampus representations are quite efficient.

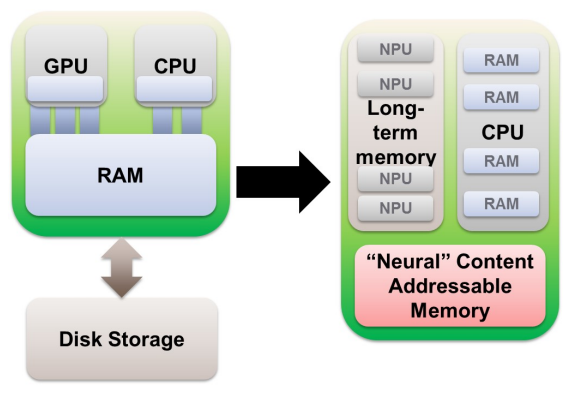

Figure 5: Potential future hybrid computing architecture leveraging neural processors (NPUs) and a neural content addressable memory.

\section{PROPOSED APPROACH TO NEURAL SUPERCOMPUTING}

Based on the arguments described above, our proposed approach to integrating neural computation into scientific computing focuses on a balanced contribution of near-term computing research leveraging existing hardware advances while embracing the potential deep impact from increased knowledge of the brain.

We suggest that pursuing SNTG logic for numerical computing is a powerful first step to take. Indeed, this is feasible near-term; given the advent of large-scale neuron-based hardware and the existing TG theoretical foundation, it is reasonable to project that a neural logic of this variety could be impactful within HPC very soon if the value could be identified.

However, we do not advocate focusing exclusively on the power of the hardware-accelerated neuron. Just as modern computer architectures are more than just a collection of transistors and logic gates, much of the brain's appeal lies in the unique and special ways that it configures its neurons. The broader computing community 
should be positioned to incorporate what is being learned during this exciting time in neuroscience research.

Finally, many of the proposed paths for machine learning in scientific computing are indeed worth pursuing. In particular, techniques such as deep learning have the potential to dramatically influence how scientific computing studies are designed and implemented at a higher level, such as smarter parameter searchers, adaptive meshing, etc. While this may not make supercomputers more powerful in an absolute sense, it could make them more useful, and that is the ultimate goal regardless of whether we are measuring in FLOPS or "NeuOPS."

Acknowledgments. This work was funded by the DOE Advanced Simulation and Computing program and Laboratory-Directed Research and Development Program at Sandia National Laboratories. Sandia National Laboratories is a multiprogram laboratory managed and operated by National Technology and Engineering Solutions of Sandia, LLC, for the U.S. Department of Energy's National Nuclear Security Administration under contract DE-NA0003525.

\section{REFERENCES}

[1] James B Aimone. 2017. Exponential scaling of neural algorithms-a future beyond Moore's Law? arXiv preprint arXiv:1705.02042 (2017)

[2] A Paul Alivisatos, Miyoung Chun, George M Church, Ralph J Greenspan, Michael L Roukes, and Rafael Yuste. 2012. The brain activity map project and the challenge of functional connectomics. Neuron 74, 6 (2012), 970-974.

[3] John R Anderson. 2009. How can the human mind occur in the physical universe? Oxford University Press.

[4] Trevor Bekolay, James Bergstra, Eric Hunsberger, Travis DeWolf, Terrence C Stewart, Daniel Rasmussen, Xuan Choo, Aaron Russell Voelker, and Chris Eliasmith. 2013. Nengo: a Python tool for building large-scale functional brain models. Frontiers in neuroinformatics 7 (2013).

[5] Markus Bläser. 2013. Fast Matrix Multiplication. Number 5 in Graduate Surveys. Theory of Computing Library. http://theoryofcomputing.org/articles/gs005/

[6] Ralph K Cavin, Paolo Lugli, and Victor V Zhirnov. 2012. Science and engineering beyond Moore's law. Proc. IEEE 100, Special Centennial Issue (2012), 1720-1749.

[7] Chris Eliasmith and Charles H Anderson. 2004. Neural engineering: Computation, representation, and dynamics in neurobiological systems. MIT press.

[8] Merrick Furst, James B. Saxe, and Michael Sipser. 1984. Parity, circuits, and the polynomial-time hierarchy. Mathematical systems theory 17, 1 (Dec. 1984), 13-27. https://doi.org/10.1007/BF01744431

[9] Alex Graves, Greg Wayne, and Ivo Danihelka. 2014. Neural turing machines. arXiv preprint arXiv:1410.5401 (2014).

[10] Jennifer Hasler and Bo Marr. 2013. Finding a roadmap to achieve large neuromorphic hardware systems. Frontiers in neuroscience 7 (2013).

[11] John J Hopfield. 1982. Neural networks and physical systems with emergent collective computational abilities. Proceedings of the national academy of sciences 79, 8 (1982), 2554-2558

[12] Giacomo Indiveri, Bernabé Linares-Barranco, Tara Julia Hamilton, André Van Schaik, Ralph Etienne-Cummings, Tobi Delbruck, Shih-Chii Liu, Piotr Dudek, Philipp Häfliger, Sylvie Renaud, et al. 2011. Neuromorphic silicon neuron circuits. Frontiers in neuroscience 5 (2011), 73.

[13] Conrad D James, James B Aimone, Nadine E Miner, Craig M Vineyard, Fredrick H Rothganger, Kristofor D Carlson, Samuel A Mulder, Timothy J Draelos, Aleksandra Faust, Matthew J Marinella, et al. 2017. A historical survey of algorithms and hardware architectures for neural-inspired and neuromorphic computing applications. Biologically Inspired Cognitive Architectures (2017)

[14] Pentti Kanerva. 1988. Sparse distributed memory. MIT press.

[15] Muhammad Mukaram Khan, David R Lester, Luis A Plana, A Rast, Xin Jin, Eustace Painkras, and Stephen B Furber. 2008. SpiNNaker: mapping neural networks onto a massively-parallel chip multiprocessor. In Neural Networks, 2008. IfCNN 2008.(IEEE World Congress on Computational Intelligence). IEEE International foint Conference on. Ieee, 2849-2856.

[16] Xavier Lagorce and Ryad Benosman. 2015. Stick: spike time interval computational kernel, a framework for general purpose computation using neurons, precise timing, delays, and synchrony. Neural computation (2015).

[17] John E Laird. 2012. The Soar cognitive architecture. MIT press.

[18] Yann LeCun, Yoshua Bengio, and Geoffrey Hinton. 2015. Deep learning. Nature 521, 7553 (2015), 436-444.
[19] Robert A. Legenstein and Wolfgang Maass. 2000. Foundations for a Circuit Complexity Theory of Sensory Processing. In Advances in Neural Information Processing Systems 13, Papers from Neural Information Processing Systems (NIPS) 2000, Denver, CO, USA, Todd K. Leen, Thomas G. Dietterich, and Volker Tresp (Eds.). MIT Press, 259-265. http://papers.nips.cc/paper/ 1910-foundations-for-a-circuit-complexity-theory-of-sensory-processing

[20] Wolfgang Maass. 1994. On the Computational Complexity of Networks of Spiking Neurons. In Proceedings of the 7th International Conference on Neural Information Processing Systems (NIPS'94). MIT Press, Cambridge, MA, USA, 183190. http://dl.acm.org/citation.cfm?id=2998687.2998710

[21] Wolfgang Maass. 1996. Lower Bounds for the Computational Power of Networks of Spiking Neurons. Neural Computation 8, 1 (1996), 1-40. https://doi.org/10. 1162/neco.1996.8.1.1 arXiv:http://dx.doi.org/10.1162/neco.1996.8.1.1

[22] Henry Markram. 2012. The human brain project. Scientific American 306, 6 (2012), 50-55.

[23] David Marr, David Willshaw, and Bruce McNaughton. 1991. Simple memory: a theory for archicortex. In From the Retina to the Neocortex. Springer, 59-128.

[24] James L McClelland, Bruce L McNaughton, and Randall C O'reilly. 1995. Why there are complementary learning systems in the hippocampus and neocortex: insights from the successes and failures of connectionist models of learning and memory. Psychological review 102, 3 (1995), 419.

[25] Paul A Merolla, John V Arthur, Rodrigo Alvarez-Icaza, Andrew S Cassidy, Jun Sawada, Filipp Akopyan, Bryan L Jackson, Nabil Imam, Chen Guo, Yutaka Nakamura, et al. 2014. A million spiking-neuron integrated circuit with a scalable communication network and interface. Science 345, 6197 (2014), 668-673.

[26] Kostas Pagiamtzis and Ali Sheikholeslami. 2006. Content-addressable memory (CAM) circuits and architectures: A tutorial and survey. IEEE fournal of SolidState Circuits 41, 3 (2006), 712-727.

[27] Ojas Parekh, Cynthia A. Phillips, Conrad D. James, and James B. Aimone. 2017. Constant Depth and Subcubic Size Threshold Circuits for Matrix Multiplication. (2017). In preparation.

[28] Catherine D Schuman, Thomas E Potok, Robert M Patton, J Douglas Birdwell, Mark E Dean, Garrett S Rose, and James S Plank. 2017. A Survey of Neuromorphic Computing and Neural Networks in Hardware. arXiv preprint arXiv:1705.06963 (2017).

[29] William Severa, Ojas Parekh, Kristofor D Carlson, Conrad D James, and James B Aimone. 2016. Spiking network algorithms for scientific computing. In Rebooting Computing (ICRC), IEEE International Conference on. IEEE, 1-8.

[30] Jiří Śíma and Pekka Orponen. 2003. General-Purpose Computation with Neural Networks: A Survey of Complexity Theoretic Results. Neural Computation 15, 12 (Dec. 2003), 2727-2778. https://doi.org/10.1162/089976603322518731

[31] Jon S Simons and Hugo J Spiers. 2003. Prefrontal and medial temporal lobe interactions in long-term memory. Nature Reviews Neuroscience 4, 8 (2003), 637-648.

[32] Kai-Yeung Siu, Vwani Roychowdhury, and Thomas Kailath. 1995. Discrete neural computation: a theoretical foundation. Prentice-Hall, Inc.

[33] Kai-Yeung Siu, Vwani Roychowdhury, and Thomas Kailath. 1995. Discrete Neural Computation: A Theoretical Foundation. Prentice-Hall, Inc., Upper Saddle River, NJ, USA.

[34] Larry R Squire. 1992. Memory and the hippocampus: a synthesis from findings with rats, monkeys, and humans. Psychological review 99, 2 (1992), 195.

[35] Prof Volker Strassen. 1969. Gaussian elimination is not optimal. Numer. Math. 13, 4 (Aug. 1969), 354-356. https://doi.org/10.1007/BF02165411

[36] Alan M Turing. 1950. Computing machinery and intelligence. Mind 59, 236 (1950), 433-460.

[37] Kei Uchizawa, Rodney J. Douglas, and Wolfgang Maass. 2006. Energy Complexity and Entropy of Threshold Circuits. In Automata, Languages and Programming, 33rd International Colloquium, ICALP 2006, Venice, Italy, July 10-14, 2006, Proceedings, Part I (Lecture Notes in Computer Science), Michele Bugliesi, Bart Preneel, Vladimiro Sassone, and Ingo Wegener (Eds.), Vol. 4051. Springer, 631-642. https://doi.org/10.1007/11786986_55

[38] Kei Uchizawa, Rodney J. Douglas, and Wolfgang Maass. 2006. On the Computational Power of Threshold Circuits with Sparse Activity. Neural Computation 18, 12 (2006), 2994-3008. https://doi.org/10.1162/neco.2006.18.12.2994

[39] Kei Uchizawa, Eiji Takimoto, and Takao Nishizeki. 2011. Size-energy tradeoffs for unate circuits computing symmetric Boolean functions. Theor. Comput. Sci. 412, 8-10 (2011), 773-782. https://doi.org/10.1016/j.tcs.2010.11.022

[40] Craig M Vineyard, Stephen J Verzi, Conrad D James, and James B Aimone. 2016. Quantifying neural information content: A case study of the impact of hippocampal adult neurogenesis. In Neural Networks (IFCNN), 2016 International foint Conference on. IEEE, 5181-5188.

[41] John Von Neumann. 2012. The computer and the brain. Yale University Press.

[42] Virginia Williams. 2014. Multiplying matrices in $O\left(n^{2.373}\right)$ time. http://theory. stanford.edu/ virgi/. (2014). online, accessed May 20, 2016.

[43] Andrew C. C. Yao. 1985. Separating the polynomial-time hierarchy by oracles. In , 26th Annual Symposium on Foundations of Computer Science, 1985. 1-10. https://doi.org/10.1109/SFCS.1985.49 